\title{
Vaccination as a trade-off between risks
}

\author{
David Crainich \\ CNRS (LEM, UMR 9221) and IÉSEG School of Management, France \\ Louis Eeckhoudt \\ IÉSEG School of Management and LEM (UMR 9221), France
}

Mario Menegatti

Dipartimento di Scienze Economiche e Aziendali, Università di Parma, Parma, Italy

\begin{abstract}
While it reduces the probability of facing a primary (or vaccine-preventable) disease, vaccination may also introduce the risk of facing vaccine induced side effects or secondary diseases. In this paper, we address the link between this feature of vaccination and attitudes toward risk. Risk aversion is shown to increase the propensity to vaccinate when the primary disease is lethal or when the risks of primary disease and of side effects are faced in different periods. When the primary disease is non-lethal and it may occur together with side effects, we show how the effect of risk aversion is affected by the probabilities and severities of each disease.
\end{abstract}

Keywords : Vaccination; self-protection; risk substitution; risk aversion.

JEL classification: D81; 110

\section{Introduction}

The promotion of vaccination was at the heart of the Global Vaccine Action Plan (GVAP) launched by 194 countries of the World Health Organization in 2012. The objective of the program is to prevent billions of death through the strengthening of vaccination policies. However, the intermediate progress towards this objective are insufficient: "In 2015, more than 19 million children missed out the basic immunization and overall global immunization coverage has stagnated" (World Health Organization, 2017). Furthermore, still according to the World Health Organization statistics, the estimated death from measles was 134200 in 2015 while influenza was believed to result in about 250 000 to 500000 deaths in 2016.

The barriers in improving global health through vaccination differ across country types. In low income countries, they mostly result from the access to vaccination and could thus be addressed by the development of immunization systems (Rainey et al. (2011)). In high income countries, these barriers are of another nature. In a recent theoretical and empirical study based on German flu vaccination data, Nuscheler and Roeder (2016) show the effects of time preferences (future-biased individuals being more vaccination prone) and of information on vaccination decisions. Using Japanese data, Tsutsui et al. (2012) also highlight that flu vaccination decisions are affected by the perceived probability of infection, by the attitude towards risk and by the rate of preference for the present. The effect of several socioeconomic variables on vaccination intentions and on vaccination behavior during the 2009-2010 H1N1 pandemic in the United States are addressed in Maurer (2015). The analysis 
shows that education and insurance coverage are positively associated with both vaccination intentions and vaccine uptake.

Few theoretical analysis have been provided yet in order to explain low-vaccination rates. Brito et al. (1991) examine public policies compelling vaccination in order to overcome the free-rider problem resulting from the positive externality of vaccination. They show that the free choice dominates compulsory vaccination for a general class of models. Geoffard and Philipson (1997) provide a rationale for the inability of vaccination to achieve - whether through private markets or public health interventions - the eradication of diseases. According to these authors, low disease prevalence achieved for instance through price subsidization or mandatory program reduce the vaccination incentives for individuals not yet vaccinated or outside the program.

The decision to vaccinate (or not) shares many points in common with the choice of a self-protection level, the analysis of which started with Ehrlich and Becker's fundamental contribution (1972). The most obvious link between the two problems is that for both of them the decision maker's objective is to reduce the probability of occurrence of an undesirable event. However there are also important differences. While the benefits of vaccination and those of self-protection are similar, the nature of some costs may be quite different. In the traditional Ehrlich and Becker's analysis the cost of selfprotection activity is known with certainty and paid in all states of the world. If vaccination is not totally subsidized, this feature for the decision maker is also present in the case of vaccination. However for vaccination there is another cost that may be much more important: while it reduces the probability of the primary disease, vaccination may induce other new risks.

We address this barrier to vaccination in our paper. A well-known aspect of vaccination is that it may induce side effects. Medical literature showed that, in some specific cases, the possible side effects may be severe and become a kind of secondary disease (see, for instance, Murphy et al., 2001 and Patel et al., 2011 about the case of rotavirus vaccines which was suspected to increase the probability of intussusception). ${ }^{1}$ Also in the general case where possible side effects are of limited size, however, the risk of their occurrence may be very significant in determining people choice to not vaccinate. Strong evidence on this has been obtained with reference to vaccination against both severe diseases (e.g. Tsuchiya et al., 2016 for mumps vaccination and Thompson et al. 2017 for HPV vaccination) and common diseases (e.g. Freimuth et al. 2017; Smith, 2017 for the case of influenza vaccination). Moreover, the recent increase in the perceived severity of vaccine risks due to false beliefs caused by controversies and misinformation in social network and non-scientific websites is well-documented by the literature and has a significant effect on people decision about vaccination (e.g. Fadda et al. 2015 and Basch et al., 2017).

Starting from this evidence, we study in this paper the decision to vaccinate or not as a trade-off between the risk of the primary disease and the risk of incurring in side effects (or secondary disease). Specifically, we analyze how the vaccination choice made by the decision maker is influenced by his degree of risk aversion. We will distinguish some different cases. First, as in most of the self-protection literature, we will assume a mono-periodic framework for the vaccination decision: the primary disease and the secondary risk are resolved in the same period. In this context there is thus no distinction between short term and long term in diseases effects. We show that, in mono-periodic

\footnotetext{
${ }^{1}$ Cases of vaccination programs suspended for suspected relationships with an increased probability of contracting severe diseases are also reported for an influenza vaccination in the United States in 1976 and for hepatitis B vaccination in France in 1998.
} 
case, two cases are to be analyzed: either one vaccinates against a lethal disease and then the degree of risk aversion has a clear implication for the decision or one vaccinates against a non-lethal disease and the impact of risk aversion becomes ambiguous because it is related to the specific characteristics of both vaccine preventable disease and vaccine induced risk. The third case analyzed considers instead a two-period framework, ${ }^{2}$ where the possible side effects or secondary disease occurs before the possible occurrence of the primary disease. This is the case characterizing short-term side effects, which are typically of limited size and disappear in the long term, when the risk of contraction of the primary disease arises. ${ }^{3}$ We show that, in this case, risk aversion has clear effects on vaccination decision.

Lastly, in a different direction, our results complement the findings by Nuscheler and Roeder (2016). While the latter also consider the potential side effects of vaccination, their model differ from ours in several important aspects: they assume that vaccination rules out infection (while it only lowers its probability in ours) and - more importantly - that agent make decisions according to Yaari's (1987) dual theory model (while we assume expected utility maximizers individuals). In the expected utility model, we distinguish the cases under which a clear role of risk aversion can be derived and those under which it is not possible.

Our paper is also related to other models in health economics. Courbage and Rey (2010), Menegatti (2014) and Brianti et al. (2017) study prevention in a health framework. All these works, however, introduce specific effects of prevention on the health dimension of agents' utility but do not consider the cost of prevention as a risk of incurring in side effects or secondary diseases. The seminal paper by Pauker and Kessirer (1975) and, more recently, Bleichrodt et al. (2003), Felder and Mayrhofer (2014) and Courbage and Rey (2016) analyze instead models of risky treatment decisions. These models, however, examine an action which increases the level of the health status in case of disease, leaving the probability of contracting the disease unchanged. On the contrary, our analysis considers a case where the action to be possibly made reduces the probability of contracting the disease. ${ }^{4}$

Our paper is organized as follows. The notations and assumptions of our model are presented in Section 2. In Section 3 we expose our results for the case of one-period framework and vaccination against a lethal disease. Section 4 presents the results in case of one-period framework and vaccination against a non-lethal disease. Section 5 analyses the case of two-period framework. Finally Section 6 concludes.

\section{Assumptions and notations}

We model a vaccination decision made by expected utility maximizing decision makers who are exposed to a vaccine preventable primary disease (henceforth "disease 1 ") that occurs with a probability $p$. In case disease 1 is contracted, their health status deteriorates from $\mathrm{H}_{0}$ (which denotes perfect health) to $H_{0}-M_{1}$ ( $M_{1}$ denoting the severity of disease 1$)$. Vaccination reduces the probability of developing disease 1 . We denote by $\Delta$ the reduction in the probability of disease 1 in case of

\footnotetext{
${ }^{2}$ Starting from Menegatti (2009), the two-period framework is alternatively used in the literature on selfprotection against financial risks.

${ }^{3}$ This is also the typical case of vaccines against influenza which usually have short-term side effects and are distributed before the expected period of contagion.

${ }^{4}$ In fact, Pauker and Kassirer's treatment is similar to the self-insurance instrument while vaccination acts as self-protection.
} 
vaccination (this probability becomes $p-\Delta$ ). ${ }^{5}$ Note that this implies that parameter $\Delta$ is a measure of the efficiency of the vaccination. Vaccination is supposed to have no financial cost but potentially introduces vaccine induced sides effects or secondary disease (henceforth "disease 2") which occurs with a probability q and with severity $M_{2}$. Since it is plausible that the primary disease has stronger effects than disease 2 we assume that $M_{1}>M_{2}$. The effects of the two diseases are supposed to be additive. Finally, the agent is facing a discrete choice: no vaccination vs vaccination. ${ }^{6}$

In next sections, we examine the impact of risk aversion on the decision to vaccinate. It is straightforward to show that the higher the efficiency of vaccination, measured by $\Delta$, the higher the propensity to vaccinate. We first compute the threshold value of the efficiency of vaccination (i.e. the value of $\Delta$ such that an individual is indifferent between vaccinating or not). A high threshold value indicates a low propensity to vaccinate (a high efficiency of vaccination is required in order to give the incentive to perform it). In order to evaluate the effects of risk aversion on the vaccination decision, we determine whether the threshold value $\Delta$ of risk averse individuals is higher than that of risk neutral ones.

Three cases will be considered: a) the case where disease 1 and disease 2 may occur in the same unique period (one-period framework) and disease 1 is lethal; $b$ ) the one-period case when disease 1 is nonlethal; c) the case where there are two periods (two-period framework) and disease 2 may occur in the first period (short term) while disease 1 may occur in the second period (long term).

\section{One-period framework and vaccination against a lethal disease.}

We first consider the one-period framework and the case where the primary disease may potentially lead to death. In this case disease 2 may occur only in the case where disease 1 does not occur since when the disease 1 occurs the disease leads to death. In such a situation the decision maker is exposed to the distributions without (Fig 1a.) and with (Fig 1b.) vaccination shown below.

Figure 1. The choice between vaccination and no vaccination in case of lethal disease.

No vaccination

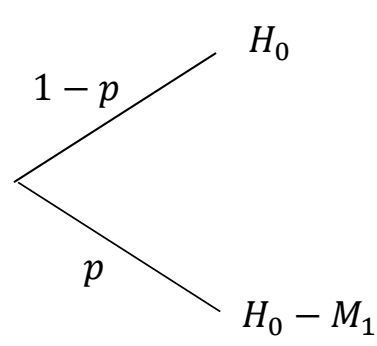

Vaccination

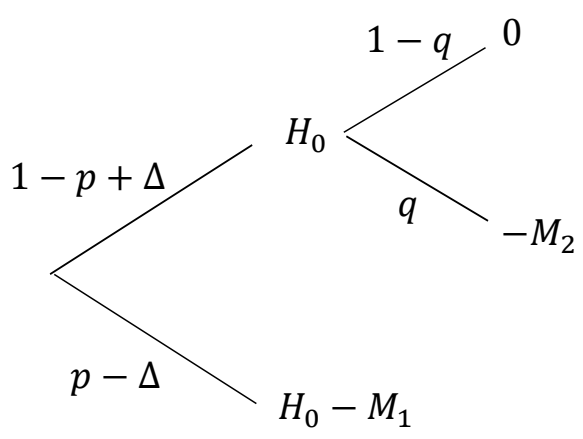

Fig 1a.

Fig $1 b$.

\footnotetext{
${ }^{5}$ A special case of this reduction, where $p=\Delta$, is considered in the paper by Nuscheler and Roeder (2016), since they study eradication of the primary disease.

${ }^{6}$ This is another and less fundamental difference between vaccination and the self-protection literature. In the latter, the decision maker has to select a level of self-protection while for vaccination the decision maker may face a binary decision (vaccinate or not) or decisions involving a finite number of consequences (depending for instance on the use of vaccine adjuvants).
} 
The threshold value of the efficiency of vaccination for risk averse individuals $\left(\Delta^{a}\right)$ is defined by the following equality:

$$
\begin{aligned}
(1-p) u\left(H_{0}\right)+p u\left(H_{0}-M_{1}\right) & \\
& =\left(1-p+\Delta^{a}\right)\left[(1-q) u\left(H_{0}\right)+q u\left(H_{0}-M_{2}\right)\right]+\left(p-\Delta^{a}\right) u\left(H_{0}-M_{1}\right)
\end{aligned}
$$

It follows that:

$$
\Delta^{a}\left[(1-q) u\left(H_{0}\right)+q u\left(H_{0}-M_{2}\right)-u\left(H_{0}-M_{1}\right)\right]=(1-p) q\left[u\left(H_{0}\right)-u\left(H_{0}-M_{2}\right)\right]
$$

The left-hand side of the equality sign is the expected benefit of vaccination while the right-hand side is its expected cost.

Rearranging the terms, the value of $\Delta^{a}$ is given by:

$$
\Delta^{a}=\frac{(1-p) q\left[u\left(H_{0}\right)-u\left(H_{0}-M_{2}\right)\right]}{u\left(H_{0}\right)-u\left(H_{0}-M_{1}\right)-q\left[u\left(H_{0}\right)-u\left(H_{0}-M_{2}\right)\right]}
$$

Now using the mean value theorem, we have:

$u\left(H_{0}\right)-u\left(H_{0}-M_{2}\right)=M_{2} u^{\prime}\left(\alpha_{2}\right)$ with $H_{0}-M_{2}<\alpha_{2}<H_{0}$

$u\left(H_{0}\right)-u\left(H_{0}-M_{1}\right)=M_{1} u^{\prime}\left(\alpha_{1}\right)$ with $H_{0}-M_{1}<\alpha_{1}<H_{0}$

so that the threshold value of the efficiency of vaccination becomes:

$$
\Delta^{a}=\frac{(1-p) q M_{2} u^{\prime}\left(\alpha_{2}\right)}{M_{1} u^{\prime}\left(\alpha_{1}\right)-q M_{2} u^{\prime}\left(\alpha_{2}\right)}=\frac{(1-p) q M_{2}}{M_{1} \frac{u\left(\alpha_{1}\right)}{u \prime\left(\alpha_{2}\right)}-q M_{2}}
$$

For risk neutral decision makers (whose marginal utility of health is constant; $u^{\prime}\left(\alpha_{1}\right)=u^{\prime}\left(\alpha_{2}\right)$ ), the threshold value of the efficiency of vaccination (denoted by $\Delta^{n}$ ) is given by: ${ }^{7}$

$$
\Delta^{n}=\frac{(1-p) q M_{2}}{M_{1}-q M_{2}}
$$

Now, given Equations (2) and (3), since $M_{1}>M_{2}$, it follows from risk aversion that $u^{\prime}\left(\alpha_{1}\right)>u^{\prime}\left(\alpha_{2}\right)$. As a result one obtains that $\Delta^{a}<\Delta^{n}$ so that risk averse decision makers are more vaccination prone. Risk averse individuals appreciate vaccination in this case for two reasons: 1 ) it reduces the probability associated to the worst outcome $\left.\left(H_{0}-M_{1}\right) ; 2\right)$ it introduces an intermediate outcome $\left(H_{0}-M_{2}\right)$ between the best $\left(H_{0}\right)$ and the worst $\left(H_{0}-M_{1}\right)$ ones. In other words, vaccination reduces the riskiness of the distribution.

\footnotetext{
${ }^{7}$ Notice that, since the probability of contracting the disease in case of vaccination cannot be negative, we need $p-\Delta \geq 0$. Starting from the value of $\Delta^{n}$, simple computations show that this requires $p M_{1} \geq q M_{2}$, meaning that the expected cost of the primary disease must be larger than the expected cost of the secondary disease. The same constraint holds in next section.
} 
The result obtained for the comparison between a risk neutral decision maker and a risk averse one can be easily extended to a comparison between two risk averse decision makers with one being more risk averse than the other one.

Let's consider in Figure 2 the utility function of $u$, who is risk averse. In this figure we also have the more concave utility function of $v$ (in discontinuous traits) who is more risk averse than $u$. Since utility is defined up to a linear transformation we can always manage to have

$$
u\left(H_{0}-M_{1}\right)=v\left(H_{0}-M_{1}\right)
$$

and

$$
u\left(H_{0}\right)=v\left(H_{0}\right)
$$

These two conditions imply

$$
u\left(H_{0}-M_{1}\right)-u\left(H_{0}\right)=v\left(H_{0}-M_{1}\right)-v\left(H_{0}\right)
$$

Given our assumptions we have that the value of $\Delta$ for $u$ is given by $\Delta^{a}$ in Equation (1), while the same value for $v$ is given by

$$
\hat{\Delta}^{a}=\frac{(1-p) q\left[v\left(H_{0}\right)-v\left(H_{0}-M_{2}\right)\right]}{v\left(H_{0}\right)-v\left(H_{0}-M_{1}\right)-q\left[v\left(H_{0}\right)-v\left(H_{0}-M_{2}\right)\right]}
$$

It is easy to see from Figure 2 that:

$$
v\left(H_{0}\right)-v\left(H_{0}-M_{2}\right)=a^{\prime} b<a b=u\left(H_{0}\right)-u\left(H_{0}-M_{2}\right)
$$

This together with Equation (4) implies that $\widehat{\Delta}^{a}$ has a smaller numerator and a larger denominator than $\Delta^{a}$, implying in turn $\widehat{\Delta}^{a}<\Delta^{a}$. So, the more risk averse agent is more vaccination prone than the less risk averse one.

Figure 2. Increase in the degree of risk aversion and the propensity to vaccinate.

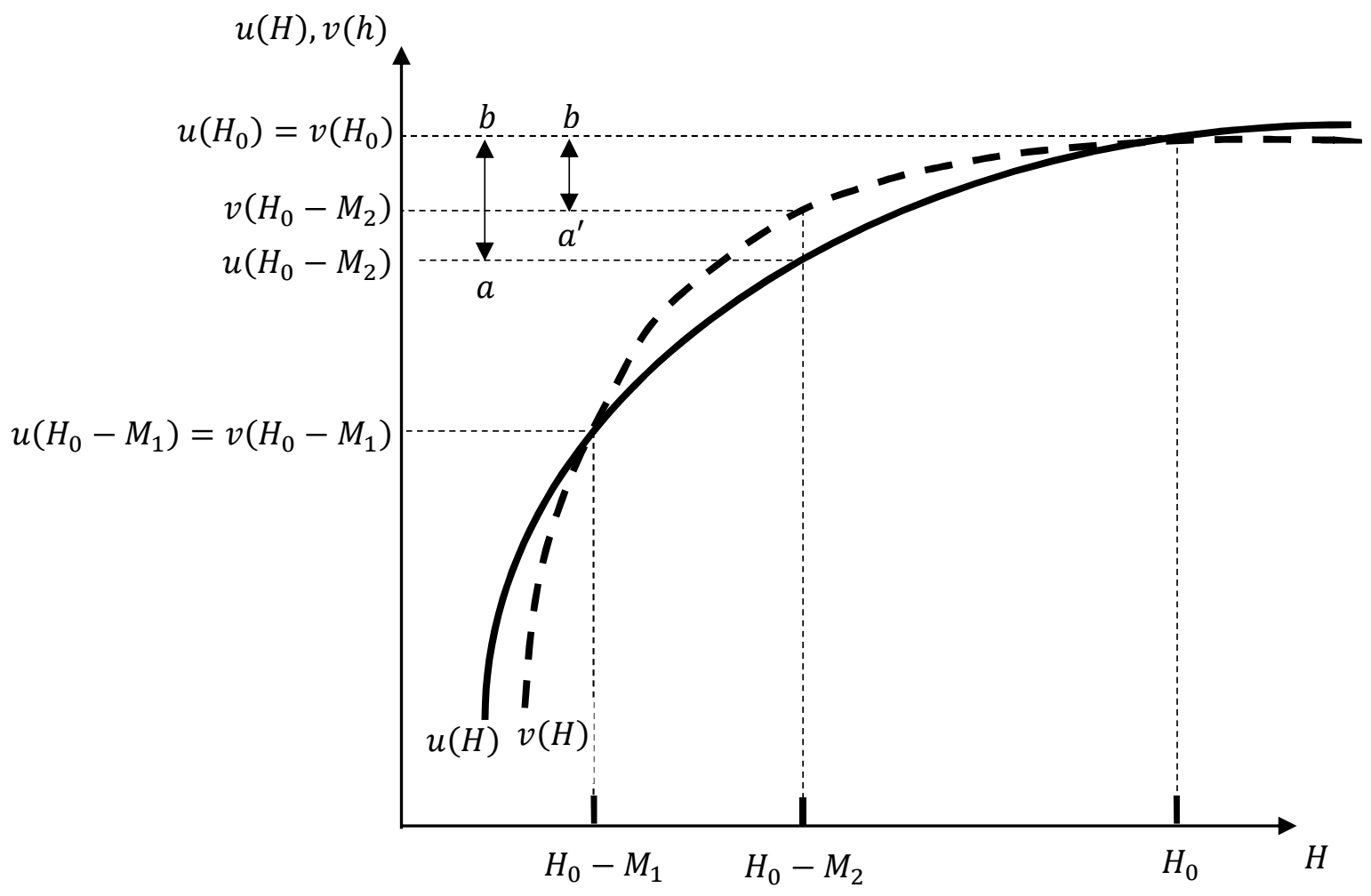




\section{One-period framework and vaccination against a non-lethal disease}

Let us assume now that, in the one-period framework, disease 1 is non-lethal, which implies that the disease 2 potentially appears both without the primary disease and with it. In such a situation, the individual is exposed to the following distributions without (Fig 3a.) and with (Fig 3b.) vaccination:

Figure 3. The choice between vaccination and no vaccination in case of non-lethal disease.

No vaccination

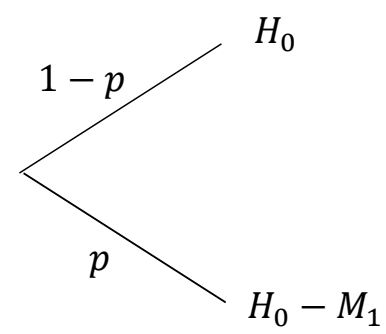

Fig 3a.

\section{Vaccination}

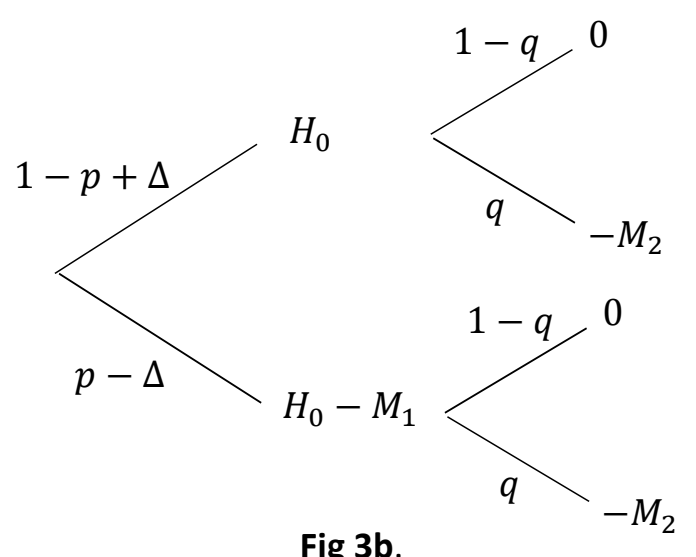

Fig 3b.

The threshold value of the efficiency of vaccination for risk averse individuals $\left(\Delta^{a}\right)$ is defined by the following equality:

$$
\begin{gathered}
(1-p) u\left(H_{0}\right)+p u\left(H_{0}-M_{1}\right)=\left(1-p+\Delta^{a}\right)\left[(1-q) u\left(H_{0}\right)+q u\left(H_{0}-M_{2}\right)\right] \\
+\left(p-\Delta^{a}\right)\left[(1-q) u\left(H_{0}-M_{1}\right)+q u\left(H_{0}-M_{2}-M_{1}\right)\right]
\end{gathered}
$$

It follows that

$$
\begin{aligned}
& \Delta^{a}\left[(1-q)\left[u\left(H_{0}\right)-u\left(H_{0}-M_{1}\right)\right]+q\left[u\left(H_{0}-M_{2}\right)-u\left(H_{0}-M_{1}-M_{2}\right)\right]\right] \\
& \quad=q\left((1-p)\left[u\left(H_{0}\right)-u\left(H_{0}-M_{2}\right)\right]+p\left[u\left(H_{0}-M_{1}\right)-u\left(H_{0}-M_{1}-M_{2}\right)\right]\right)
\end{aligned}
$$

As in Section 3, the left-hand side of the equality sign is the expected benefit of vaccination while the right-hand side is its expected cost. The former is a weighted average of the benefit of vaccination in the absence of secondary disease $\left(\Delta\left[u\left(H_{0}\right)-u\left(H_{0}-M_{1}\right)\right]\right)$ and in its presence $\left(\Delta\left[u\left(H_{0}-M_{2}\right)-\right.\right.$ $\left.\left.u\left(H_{0}-M_{1}-M_{2}\right)\right]\right)$. The latter is the weighted average of the impact of disease 2 in the absence of disease $1\left(u\left(H_{0}\right)-u\left(H_{0}-M_{2}\right)\right)$ and in its presence $\left(u\left(H_{0}-M_{1}\right)-u\left(H_{0}-M_{1}-M_{2}\right)\right)$.

Rearranging the terms, the value of $\Delta^{a}$ is given by:

$$
\Delta^{a}=\frac{q\left((1-p)\left[u\left(H_{0}\right)-u\left(H_{0}-M_{2}\right)\right]+p\left[u\left(H_{0}-M_{1}\right)-u\left(H_{0}-M_{1}-M_{2}\right)\right]\right)}{(1-q)\left[u\left(H_{0}\right)-u\left(H_{0}-M_{1}\right)\right]+q\left[u\left(H_{0}-M_{2}\right)-u\left(H_{0}-M_{1}-M_{2}\right)\right]}
$$

Simple computations also show that the threshold efficiency for risk neutral individuals (denoted by $\left.\Delta^{n}\right)$ is given by: 


$$
\Delta^{n}=\frac{q M_{2}}{M_{1}}
$$

Now, using the mean value theorem, we have:

$u\left(H_{0}-M_{1}\right)-u\left(H_{0}-M_{1}-M_{2}\right)=M_{2} u^{\prime}\left(\alpha_{3}\right)$ with $H_{0}-M_{1}-M_{2}<\alpha_{3}<H_{0}-M_{1}$

$u\left(H_{0}-M_{2}\right)-u\left(H_{0}-M_{1}-M_{2}\right)=M_{1} u^{\prime}\left(\alpha_{4}\right)$ with $H_{0}-M_{1}-M_{2}<\alpha_{4}<H_{0}-M_{2}$

Using these definitions of $\alpha_{3}$ and $\alpha_{4}$ as well as those of $\alpha_{1}$ and $\alpha_{2}$ provided in Section 3, the threshold value of the efficiency of vaccination for risk averse agents becomes:

$$
\Delta^{a}=\frac{q M_{2}\left((1-p) u^{\prime}\left(\alpha_{2}\right)+p u^{\prime}\left(\alpha_{3}\right)\right)}{M_{1}\left((1-q) u^{\prime}\left(\alpha_{1}\right)+q u^{\prime}\left(\alpha_{4}\right)\right)}
$$

As a result, the comparison between $\Delta^{a}$ and $\Delta^{n}$ also shows that the risk averse decision makers are more vaccination prone than the risk neutral decision makers $\left(\Delta^{a}<\Delta^{n}\right)$ if:

$$
(1-p) u^{\prime}\left(\alpha_{2}\right)+p u^{\prime}\left(\alpha_{3}\right)<(1-q) u^{\prime}\left(\alpha_{1}\right)+q u^{\prime}\left(\alpha_{4}\right)
$$

and they are less vaccination prone $\left(\Delta^{a}>\Delta^{n}\right)$ if the opposite inequality holds. It is thus easy to see that in case of non-lethal disease we do not have a clear direction for the comparison between the choices of risk averse and risk neutral agents. In particular the relative values of $\alpha_{1}, \alpha_{2}, \alpha_{3}$ and $\alpha_{4}$ are represented in Figure 4 below.

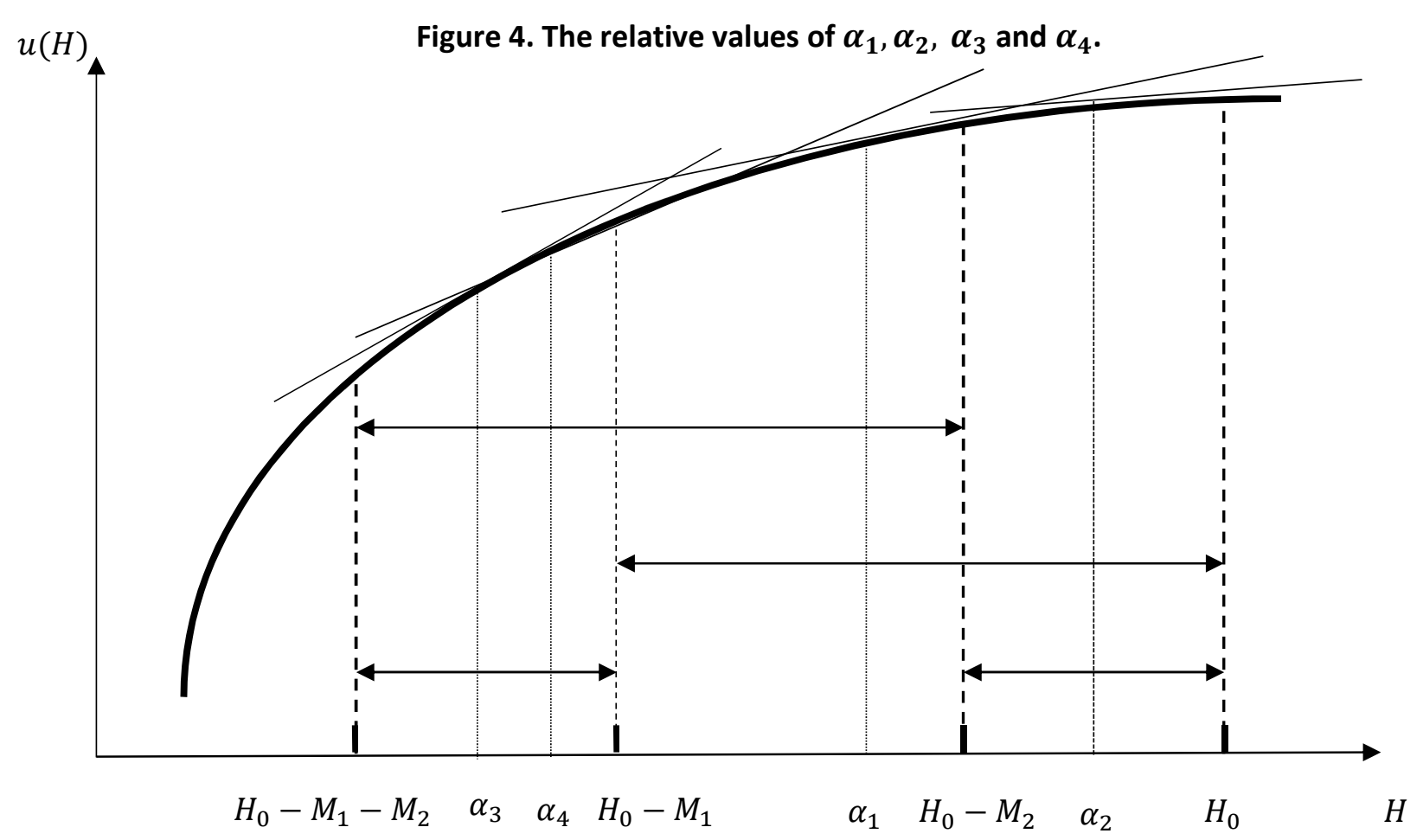

The relative values of $\alpha_{1}, \alpha_{2}, \alpha_{3}$ and $\alpha_{4}$ (see (2), (3), (5) and (6)) imply that $u^{\prime}\left(\alpha_{3}\right)>u^{\prime}\left(\alpha_{1}\right)$ and $u^{\prime}\left(\alpha_{4}\right)>u^{\prime}\left(\alpha_{2}\right)$. This means that, for instance, when $p$ is sufficiently low and $q$ is sufficiently high, Inequality (7) holds and the risk averse individuals are more vaccination prone than the risk neutral 
individuals. On the contrary when $p$ is sufficiently high and $q$ is sufficiently low, the opposite of Inequality (7) holds and the risk averse individuals are less vaccination prone than the risk neutral individuals.

Another simple example where the risk averse decision maker can be either more or less prone to vaccinate than the risk neutral agent may be obtained under the assumption that the risk averse decision maker has a quadratic utility function, i.e. when $u(x)=c x-\frac{x^{2}}{2}$ where $c>H_{0}$. Under this assumption we have: ${ }^{8}$

$$
\Delta^{a}=\frac{q M_{2}\left[p\left(c-\frac{1}{2}\left(2 H_{0}-2 M_{1}-M_{2}\right)\right)+(1-p)\left(c-\frac{1}{2}\left(2 H_{0}-M_{2}\right)\right)\right]}{M_{1}\left[q\left(c-\frac{1}{2}\left(2 H_{0}-M_{1}-2 M_{2}\right)\right)+(1-q)\left(c-\frac{1}{2}\left(2 H_{0}-M_{1}\right)\right)\right]}
$$

Comparing $\Delta^{a}$ with $\Delta^{n}$ we get, after some computations, that Inequality (5) above becomes in this case

$$
(1-2 p) M_{1}>(1-2 q) M_{2}
$$

It is easy to see that, in this case, given the values for $p$ and $q$, Inequality (7) surely holds and the risk averse decision maker is more vaccination prone than the risk neutral decision maker if $M_{1}$ is sufficiently larger than $M_{2}$. If, on the contrary, $M_{1}$ is not sufficiently large, the opposite of Inequality (7) may hold and the risk averse decision maker is less vaccination prone than the risk neutral decision maker.

Lastly, the comparison between $\Delta^{a}$ and $\Delta^{n}$ also shows the different effects of changes in probability of contracting the primary disease for the two decision makers. In particular, it is easy to see that $\frac{d \Delta^{a}}{d p}>$ 0 and $\frac{d \Delta^{n}}{d p}=0$. This different effect has a clear interpretation. An increase in $p$ implies an increase in the probability of facing the primary disease together with the secondary disease. This is significant for the risk averse decision maker, who dislikes the case where he contemporaneously suffers from both diseases, while it is not relevant for the risk neutral decision maker, who is indifferent between the two cases where the diseases occur together or separately. Consequently the expected cost of vaccination is an increasing function of $p$ for the risk averse agent while it does not depend on $p$ for the risk neutral agent.

\section{Two-period framework}

Vaccination decisions are sometimes examined in multi-period model as in Geoffard and Philipson (1997) or in Nuscheler and Roeder (2016). In this section we adopt this approach and we suppose that the side effects or the secondary disease may occur only in the short term (and their effects do not last through time) while the primary disease may occur in a subsequent period, typically the long term.

We consider, in particular, a two-period framework. In the first period the agent chooses to vaccinate or not. If he vaccinates he remains healthy with probability (1-q) and incurs side effects (disease 2 ) with probability $q$ while if he does not vaccinate he is surely healthy. On the other hand, in the second period, the agent faces the risk of developing the primary disease (disease 1 ) and the probability that this occurs is

\footnotetext{
${ }^{8}$ Note that under quadratic utility the mean value theorem ensures that $u(y)-u(z)=u^{\prime}(s)[y-z]$ where $s=$ $\frac{y+z}{2}$.
} 
$p$ in case of no vaccination and $p-\Delta$ in case of vaccination. The health state in the absence of disease $\left(H_{0}\right)$ is supposed to remain unchanged from one period to the other. We also assume, for simplicity, that the intertemporal discount factor is 1 . The risks faced by the agent in case of vaccination and of no vaccination are thus those described in Figure 5 below.

Figure 5. The choice between vaccination and no vaccination in the two-period framework.

\section{No vaccination}

period 1

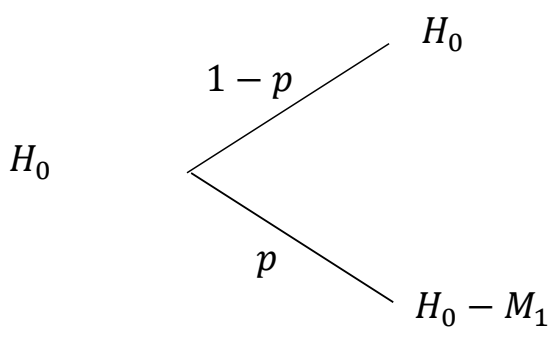

Fig $5 a$.

\section{Vaccination}

period 1

period 2
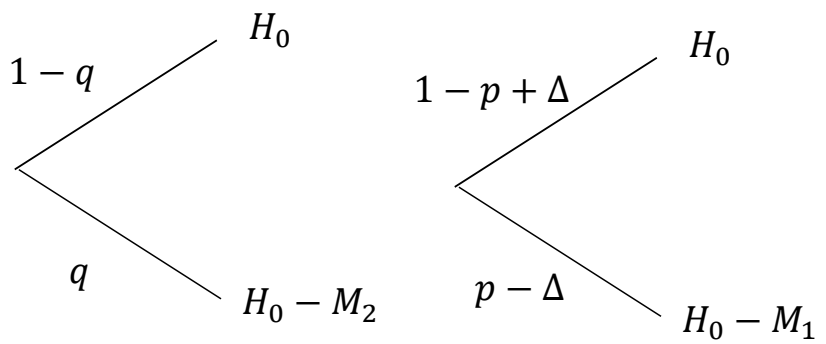

Fig 5b.

Now, following the technique exploited in sections 3 and 4, we equalize the expected utilities with and without vaccination.

$$
\begin{aligned}
u\left(H_{0}\right)+(1-p) & u\left(H_{0}\right)+p u\left(H_{0}-M_{1}\right) \\
& =(1-q) u\left(H_{0}\right)+q u\left(H_{0}-M_{2}\right)+(1-p+\Delta) u\left(H_{0}\right)+(p-\Delta) u\left(H_{0}-M_{2}\right)
\end{aligned}
$$

From the above equation, we obtain the following threshold value of the efficiency of vaccination for risk averse $\left(\Delta^{a}\right)$ and risk neutral $\left(\Delta^{n}\right)$ individuals.

$$
\begin{gathered}
\Delta^{a}=q \frac{u\left(H_{0}\right)-u\left(H_{0}-M_{2}\right)}{u\left(H_{0}\right)-u\left(H_{0}-M_{1}\right)} \\
\Delta^{n}=q \frac{M_{2}}{M_{1}}
\end{gathered}
$$

Using the mean value theorem, the threshold value of the efficiency of vaccination for risk averse individuals is given by:

$$
\Delta^{a}=q \frac{M_{2} u^{\prime}\left(\alpha_{2}\right)}{M_{1} u\left(\alpha_{1}\right)}
$$

with $H_{0}-M_{1}<\alpha_{1}<H_{0}$ and $H_{0}-M_{2}<\alpha_{2}<H_{0}$

Since $M_{1}>M_{2}$ and $u^{\prime \prime}<0$, it follows that $u^{\prime}\left(\alpha_{2}\right)<u^{\prime}\left(\alpha_{1}\right)$. It is thus straightforward to show that risk averse individuals are more vaccination prone than risk neutral ones $\left(\Delta^{a}<\Delta^{n}\right)$. 


\section{Conclusion}

The economic evaluation of vaccination programs requires the assessment of the effect of risk attitude on the propensity to vaccinate. In this paper we show that, in the case where the vaccine-preventable primary disease may occur in the same period of side effects or secondary disease possibly induced by the vaccine, the consequences of different risk attitudes depend on the type of vaccine-preventable disease considered. When individuals vaccinate against lethal diseases, the demand for vaccination increases with the degree of risk aversion. This clear-cut relationship no longer prevails when they vaccinate against non-lethal diseases. In this situation we derive some implications for the choices made by decision-makers who face different levels of probabilities and/or severities of diseases. Lastly, in the case where possible side effects are limited to the short term and may occur only before the period where the contraction of the primary disease is possible, the demand for vaccination is larger for risk averse agents, independently from the type of vaccine-preventable disease considered.

These different conclusions have a possible common interpretation. Risk aversion pushes an individual to be more vaccination prone when the risk of side effects or secondary disease is separate from the risk of contracting the vaccine-preventable disease. This happens either when the vaccine-preventable disease is lethal or when the possible side effects surely precede the primary disease and occurs since, in these situations, the risk averse agent only has a strong desire to escape the worse risk, i.e. the primary disease. On the contrary, the case where the risk of contracting a non-lethal vaccinepreventable disease and the risk of secondary disease are faced together makes it possible that the decision maker incurs the two diseases together. Since a risk averse agent strongly dislikes this event, this generates a multifaceted picture, which makes ambiguous the effect of risk aversion on the decision to vaccinate or not.

\section{References}

Basch CH, Zybert P, Reeves R, Basch CE, (2017), "What do popular YouTube ${ }^{\mathrm{TM}}$ videos say about vaccines?", Child: Care, Health and Development, forthcoming.

Bleichrodt, H, Crainich, D, Eeckhoudt, L (2003), "The effect of comorbidities on treatment decisions", Journal of Health Economics, 22(5), pp. 805-820

Boyko EJ, Koepsell TD, Perera DR, Inui TS (1987), "Risk of ulcerative colitis among former and current cigarette smokers", New England Journal of Medicine, 316, 707-710.

Brianti M, Menegatti M, Magnani M, (2017), "Optimal choice of prevention and cure under uncertainty on disease effect and cure effectiveness, Research in Economics, forthcoming, DOI: 10.1016/j.rie.2017.03.005.

Brisson M, Gay NJ, Edmunds WJ, Andrews NJ (2001), "Exposure to varicella boosts immunity to herpeszoster: implications for mass vaccination against chickenpox“, Vaccine, 20, 2500--2507.

Brito D, Sheshinski E, Intriligator M (1991), "Externalities and compulsory vaccinations", Journal of Public Economics, 45, 69-90. 
Courbage C, Rey B (2016), "Decision thresholds and changes in risk for preventive treatment", Health Economics, 25(1), 111-124.

Ehrlich I, Becker G (1972), "Market insurance, self-insurance, and self-protection", The Journal of Political Economy, 80(4), 623-648.

Fadda M, Allam A, Schultz PJ (2015) Arguments and sources on Italian online forums on childhood vaccinations: Results of a content. Vaccine 33 (51), 7152-7159.

Felder, S, Mayrhofer T (2014) "Risk preferences: consequences for test and treatment thresholds and optimal cutoffs." Medical Decision Making 34(1), 33-41.

Freimuth VS, Jamison A, Hancock G, Musa D, Hilyard K, Crouse Quinn S, (2017) "The Role of Risk Perception in Flu Vaccine Behavior among African-American and White Adults in the United States", Risk Analysis, forthcoming, DOI: 10.1111/risa.12790.

Geoffard P-Y, Philippson T (1997), “Disease Eradication: Private versus Public Vaccination”, American Economic Review, 87(1), 222-230.

Harries AD, Baird A, Rhodes J (1982), "Non-smoking: a feature of ulcerative colitis", British Medical Journal, 284, 706.

Hviid A, Stellfeld M, Wohlfahrt J, Melbye M, (2004), "Childhood Vaccination and Type 1 Diabetes", New England Journal of Medicine, 350, 1398-1404.

Maurer J (2015), "Inspecting the Mechanism - A Longitudinal Analysis of Socioeconomic Status Differences in Perceived Influenza Risks, Vaccination Intentions, and Vaccination Behaviors during the 2009-2010 Influenza Pandemic", Medical Decision Making, 36(7), 887-899.

Menegatti M (2009), "Optimal prevention and prudence in a two period model", Mathematical Social Science, 58, 393-397.

Menegatti, M. (2014). "Optimal choice on prevention and cure: A new economic analysis", The European Journal of Health Economics, 15(4), 363-372.

Murphy TV, Gargiullo PM, Massoudi MS, et al. (2001), "Intussusception among infants given an oral rotavirus vaccine", New England Journal of Medicine, 364, 564-572.

Nuscheler R, Roder K (2016), "To vaccinate or to procrastinate? That is the prevention question", Health Economics, 25(12), 1560-1581.

Patel MM, Richardson Lopez-Collada V, Mattos Bulhoes M, et al. (2011), "Intussusception risk and health benefits of rotavirus vaccination in Mexico and Brazil", New England Journal of Medicine, 364, 2283-2292.

Pauker SG, Kassirer JP (1975), "Therapeutic decision making: a cost-benefit analysis", New England Journal of Medicine, 293, 229-234. 
Rainey JJ, Watkins M, Ryman TK, Sandhu P, Bo A, Banerjee K (2011), "Reasons related to nonvaccination and under-vaccination of children in low and middle income countries: Findings from a systematic review of the published literature, 1999-2009", Vaccine, 29(46), 8215-8221.

Smith LE, Webster RK, Weinman J, Amlôt R, Yiend J, Rubin GJ (2017), "Psychological factors associated with uptake of the childhood influenza vaccine and perception of post-vaccination side-effects: A cross-sectional survey in England" Vaccine 35 (15), 1936-1945.

Thompson, E. L., Rosen, B. L., Vamos, C. A., Kadono, M., Daley, E. M. (2017). "Human Papillomavirus Vaccination: What Are the Reasons for Nonvaccination Among US Adolescents?" Journal of Adolescent Health, 61(3), 288-293.

Tsuchiya Y, Shida N, Izumi S, Ogasawara M, Kakinuma W, Tsujiuchi T, Machida K (2016) "Factors associated with mothers not vaccinating their children against mumps in Japan“, Public Health 137, 95105.

Tsutsui Y, Benzion U, Shahrabani S (2012), "Economic and behavioral factors in an individual's decision to take the influenza vaccination in Japan", The Journal of Socio-Economics, 41(5), 594-602.

World Health Organization, 2017, report of the World Health Assembly Global Vaccine Action Plan.

Yaari ME, (1987), "The dual theory of choice under risk", Econometrica 55, 95-115. 